\title{
Téoros
}

Revue de recherche en tourisme

\section{La formation en région}

Bilan et perspectives

\section{Danielle Ouellet}

Volume 16, numéro 1, printemps 1997

La région touristique de l'Estrie / Cantons-de-l'Est

URI : https://id.erudit.org/iderudit/1074928ar

DOI : https://doi.org/10.7202/1074928ar

Aller au sommaire du numéro

Éditeur(s)

Université du Québec à Montréal

ISSN

0712-8657 (imprimé)

1923-2705 (numérique)

Découvrir la revue

Citer cet article

Ouellet, D. (1997). La formation en région : bilan et perspectives. Téoros, 16(1), 50-53. https://doi.org/10.7202/1074928ar

Ce document est protégé par la loi sur le droit d'auteur. L'utilisation des services d'Érudit (y compris la reproduction) est assujettie à sa politique d'utilisation que vous pouvez consulter en ligne.

https://apropos.erudit.org/fr/usagers/politique-dutilisation/
Cet article est diffusé et préservé par Érudit.

Érudit est un consortium interuniversitaire sans but lucratif composé de l'Université de Montréal, l'Université Laval et l'Université du Québec à Montréal. Il a pour mission la promotion et la valorisation de la recherche. https://www.erudit.org/fr/ 


\section{LA FORMATION EN RÉGION}

\section{BILAN ET PERSPECTIVES}

\section{Danielle Ouellet, agente de développement - Gestion des ressources humaines et formation Tourisme Cantons-de-l'Est}

A l'ete 1993. Tourisme Estrie donnait suite aux recommandations d'un Comite' d'adaptation de la main-d'oeuvre (CAMO) en confiant à une firme de consultants le mandat d'évaluer la pertinence el la faisabilité de se doter d'un service de gestion des ressources humaines. Ce service aurait pour mission d'assister les entreprises membres dans la gestion de leurs ressources humaines et dans l'articulation de programmes concrets de formation.

Cette évaluation s'est faite à partir d'un sondage auprès de tous les membres de Tourisme Cantons-de-l'Est. Plus de 80 membres y ont répondu. Les principaux constats qui se dégagent des résultats de ce sondage sont les suivants:

- Très peu d'entreprises ont un gestionnaire dont la principale tâche est la gestion des ressources humaines:

- Elles investissent beaucoup de temps pour embaucher le personnel, en initier du nouveau et gérer les mises à pied et les départs;

- Elles semblent utiliser un processus de sélection plus ou moins efficace:

- Elles ont besoin d'améliorer leurs politiques ou procédures dans la gestion des ressources humaines:

- Elles investissent peu dans l'amélioration des compétences de leur personnel.

- Les dirigeants d'entreprises ne semblent avoir ni le temps, ni les ressources pour se doter d'outils de gestion valides et efficaces.

Un consensus semble donc se dégager à l'effet qu'il existe des besoins en matière de gestion des ressources humaines. Les répondants démontrent une certaine ouverture à recevoir du support en formation et pour le développement d'outils de gestion mais presque tous ont exprimé leur réticence devant la possibilité d'une intervention directe dans la gestion de leur entreprise. Ils se disent alors intéressés à payer pour un tel service mais seulement dans la mesure où ils en profitent personnellement. IIs favorisent done le principe de l'utilisateur payeur.

Sur la base de ces observations, Tourisme Cantons-de-l'Est décide de donner suite à la recommandation des consultants et embauche, en décembre, une personne spécialisée en gestion des ressources humaines. Voici le mandat qui lui est confié:

- Identifier des réponses concrètes aux besoins des membres, s'assurer de leur faisabilité et les mettre en oeuvre:

- Sensibiliser les membres à la nécessité de la formation;

- Offrir ou coordonner des activités de formation adaptées aux besoins de l'industrie touristique:
- Identifier les subventions disponibles et supporter les membres dans leurs démarches pour bénéficier de ces subventions.

Pour supporter la réalisation de ce mandat, on met en place un comité d'orientation constitué de représentants des différents secteurs de l'industrie.

\section{DIAGNOSTIC DE LA SITUATION}

Une première tournée de consultation est réalisée en mars 1994. En discutant avec les propriétaires et dirigeants de plus de 20 entreprises, réparties sur l'ensemble du territoire et représentant tous les secteurs, on précise les demandes du milieu. Les membres désirent:

- Des éléments de contenu concrets, facilement applicables et qui ont un effet immédiat:

- Du matériel didactique très adapté au milieu:

- Des formateurs qui connaissent l'industrie touristique en général et leur secteur d'activités en particulier:

- Certains désirent plus de la documentation que de la formation pour éviter les déplacements et les frais inutiles;

- Des outils, des méthodes et des conseils qui leur permettent de faire mieux et plus efficacement ce qu'ils font dejà;

- Des formations de courte durée (3-4 heures);

- Les coûts les plus bas possibles. 
Ils se disent réticents à changer leurs façons de faire à moins qu on leur prouve qu'il $y$ aura des rćsultats intéressants pour eux et observables à court terme.

On apprend aussi que chaque entreprise compte un petit nombre d'employés à former et ces demiers ont des besoins tress diversifies. Quand on leur parle de la possibilité de regrouper les entreprises en vue de leur offrir les services, les repondants favorisent les regroupements locaux aux regroupements régionaux. En effet, alors que les gestionnaires se disent prêts à se déplacer, ills ne veulent pas qué leurs employés en fassent autant. Plusieurs manifestent done leur intention d'aller chercher l'information et de former eux-mêmes leur personnel.

\section{LES SOLUTIONS PRÉCONISÉES}

Pour répondre à ces besoins, il est tout d'abord décidé d'aller de l'avant avec un projet de création d'une banque d'outils de formation destinés prioritairement à la petite et moyenne entreprise. Les thèmes retenus pour débuter sont:

- L'entretien des chambres;

- La réception des clients;

- La carte des vins;

- La reception d'appels:

- Le service de table.

Pour chacun de ces thèmes. on réalise un vidéo dont le scénario est élaboré par Tourisme Cantons-de-l'Est avec la collaboration de gens de l'industrie réunis au sein d'un comitét d'experts. Chaque vidéo, d'une durée variant de 10 à 15 minutes, présente des techniques de base dans le domaine traité. Un guide de l'animateur permet ensuite à l'utilisateur d'adapter le contenu du vidéo à la réalité de son entreprise et lui fournit des suggestions pour la formation des employés. Enfin, un outil est préparé pour l'usage de l'apprenti qui suivra la formation. Le tout est offert aux membres à l'intérieur d'un coffret vendu à prix forfaitaire.

A ce jour, deux coffrets sont disponibles soit L'entretien des chambres et Unclient au bout du fil. Les trois autres devraient être mis en marché au printemps 1997. Les deux premiers ont reçu un bon accueil du milieu. Plus de 30 coffrets de chacun de ces thèmes ont été vendus à des entreprises d'un peu partout au Québec. Et, à notre grande surprise. moins de la moitié de ces coffrets ont été achetés par des entreprises de la région.

Parallèlement, Tourisme Cantons-del'Est élabore un programme de formation de formateurs à l'interrieur duquel on encadre le développement de matériel de formation personnalisé pour l'entreprise et on formalise le processus de formation dit asur le tasw. Dans la première année, quatre groupes d'une dizaine de personnes suivent la formation avec succès. Les participants à ces formations développent du matériel de qualité avec l'assistance du formateur. Les plus impliqués obtiennent des résultats fort intéressants. Mais ce qui nous frappe le plus, c'est l'intérêt que manifestent toutes les personnes qui suivent le cours à structurer et à améliorer tous les types de formation qu'elles donnent par la suite au sein de l'entreprise. Et les retombées dépassent même les limites de la formation pour se généraliser à la gestion des ressources humaines. En effet, la réflexion initiée a amené certains d'entre eux à produire ou à réviser leur guide du nouvel employé, à adopter une politique d'accueil et à remettre en question leurs critères et leurs méthodes d'embauche.

Des formations de courte durće, de 4 à 8 heures chacune, sont aussi organisées. Parmi les thèmes traites, notons:

- Le nouveau code civil;

- Recrutement et mises à pied de personnel;

- Préparation de matériel publicitaire et promotionnel;

- Atelier de rédaction d'un dépliant publicitaire:

- Formation des préposés à l'accueil;

- Accueil pour les intervenants de première ligne;

- Élaboration de forfaits;

- Taxi Ambassadeur;

- Agrotourisme;

- Télémarketing.

Des cours d'anglais fonctionnel, niveaux débutant, intermédiaire et avancé sont offerts à chaque année et une chronique du bulletin TourismEstrie est réservée aux activités de formation et à différents sujets en gestion des ressources humaines.

L'ensemble de ces activités est mené en collaboration très étroite avec la Société québécoise de développement de la main-d'oeuvre (SQDM) de l'Estrie qui finance une bonne partic des operations, à commencer par la moitié du salaire de l'agente de développement. Avec le temps, le secteur tourisme se voit allouer une enveloppe budgétaire spécifique, ce qui permet d'offrir toutes nos formations à trìs bas prix et, par le fait même, d'attirer un plus grand nombre de participants. L"approche en est une de partenariat de part et d'autre. L'ATR aide la SQDM à faire une percée au niveau de l'industrie touristique, ce qui faisait partie de son plan d"action en région et la SQDM soutien les efforts de développement de $1^{4}$ ATR en collaborant à l'élaboration des projets plutôt qu'en les jugeant une fois élaborés. L'ensemble des dossiers est donc abordé avec une approche de resolution de problèmes, créant ainsi une grande ouverture pour le développement.

\section{DIFFICULTÉS ET PARADOXES}

Au départ, nous avons tenté de faire l'in= ventaire des formations, des formateurs et des outils de formation disponibles sur le marché pour répondre aux demandes des membres.

Prenons seulement l'exemple de la formation en accueil et service à la clientèle qui faisait l'objet d'une demande quasi unanime de la part de nos membres. Aucun programme formel répondant à des exigences minimales en matière de transfert d'apprentissage n'était disponible sur le marché. Plusieurs formateurs offraient bel et bien aux participants de leur livrer des trucs ou des recettes agrémentés d'exemples de situations désastreuses lors de séminaires pouvant durer de trois heures à deux jours. Il nous a toutefois été impossible d'identifier un seul produit structuré, basé sur des objectifs d'apprentissage mesurables ou observables, qui permette d'évaluer les résultats de la formation et qui utilise des méthodes pédagogiques adaptées aux besoins spécifiques des travailleurs de l'industrie touristique. 
Il nous a aussi été très difficile de trouver des formateurs d'expentience, spécialisés pour l'industrie touristique. Nous avons trouve de bons formateurs qui ne connaissent pas le tourisme. Nous avons aussi trouvé des gens qui ont beaucoup d'expérience terrain en tourisme mais qui connaissent peu ou pas la notion de transfert d'apprentissages. Le cumul des deux semble, quant à lui, être une denrite très rare.

Quant au materiel de formation, nous n'avons identifié que quelques produits en français traitant principalement de santé et sécurité au travail mais rien qui réponde vraiment à nos besoins immédiats. Nous nous sommes vite rendu compte que le très grand nombre d"intervenants, qu'on pense seulement aux nombreuses associations sectorielles, rendait extremement difficile la recherche du materiel disponible. Plusieurs nous ont répondu qu'ils avaient l'intention de développer des choses pour répondre aux demandes de leurs membres mais qu'ils devaient avant tout trouver le financement nécessaire.

Bref, nous partions presque à zéro. En dehors des grandes écoles spécialisées en tourisme, il n'y avait pour ainsi dire rien d'intéressant pour nous sur le marché privé de la formation et le développement par d'autres intervenants était trop incertain pour s'y fier à courte écheance. Nous avons donc décidé de développer nos propres programmes de formation, de former nos formateurs et de développer notre matériel de formation pour répondre localement aux besoins de nos membres.

Quand est venu le temps de mettre en marché les produits développés, nous nous sommes rendu compte que les besoins étaient plutôt généralisés par secteur d'activikés que par région. Nos vidéos se vendaient partout au Québec, mais moins que nous pensions dans notre propre région. Et nous en sommes venus à nous poser la question suivante: Pourquoi, homis le fait que personne ne l'avait fait ayant nous, une ATR devrait= elle développer du matériel spécialisé pour l'industrie de l'hôtellerie? Est-ce aux membres d'une ATR à payer pour ce développement? Et pourquoi pas? La question reste entière.
Alors que nous étions attablés à développer du matériel de formation, une autre question ne nous quittait pas l'esprit. $Y$ avait-il, quelque part au Québec, une autre association qui travaillait à développer le même type de produit que nous, traitant du même sujet, sans qu'on le sache. En effet, tout le monde peut développer des produits de formation. chacun dans son coin, et dépenser en double des montants si difficiles à trouver alors que les besoins sont si étendus. Comment faire pour être tenus au courant des initiatives de l'ensemble des intervenants de l'industrie en formation? Cette question est partiellement répondue par l'entrée en fonction, en juin 1996, du Conseil québeccois des ressources humaines en tourisme (CQRHT) qui regroupe en son sein des représentants de toutes les associations sectorielles et régionales en vue de coordonner le développement dans ce domaine. Par son intervention, le CQRHT devrait être en mesure d'assurer un développement harmonieux et durable de l'industrie de la formation en tourisme.

Le principal problème demeure toutefois au niveau du financement d"un poste en formation au sein d'une association régionale. Lors de l'embauche de l'agente de développement, en décembre 1993 , le conseil d'administration de I'ATR insistait sur la nécessité d'autofinancer le poste. Pour y arriver, on comptait d'abord sur les subventions puis sur les revenus découlant de l'organisation de formations, de la vente du matériel dếveloppé et d'activités de consultation en gestion des ressources humaines aupres des entreprises membres.

Dans les faits, comme la formation ne faisait pas vraiment partie de la culture de nos entreprises touristiques et qu'il s'est avérét extrémement difficile de recruter le nombre reguis de participants pour profiter des subventions disponibles, nous avons dû maintenir le coût d"inscription très bas. Par le fait même, les profits liés à l'organisation d'activités de formation ont été négligeables.

Les coûts de production des coffrets de formation, plus élevés que prévu, ont aussi limité la marge de profit disponible. Nous réussissons à couvrir nos frais, mais sans plus.
La consultation, quant à elle, s'est avérée une excellente source de financement dans la mesure où nous sommes capables de vendre l'idée aux membres de payer pour faire faire ce qu' ils ont toujours fait eux-mèmes. Et la tâche est de taille car, même vendu à taux préférentiel, peu nombreuses sont les entreprises touristiques qui peuvent se payer un taux horaire de consultant. Au depart, les entreprises interrogées se disaient prêtes à payer pour ce service dans la mesure où elles l'utilisaient. Mais sont-elles prêtes à utiliser les services d'un consultant pour améliorer leur gestion des ressources humaines? La question, encore aujourd'hui, reste sans réponse.

Alors que la formation est jugéce essentielle et urgente par tous les intervenants qui agissent sur le développement de l'industrie touristique, les entreprises n'en achètent que très peu et ce, peu importe la forme sous laquelle elle leur est offerte. Et comme les intervenants n'en achètent pas, il est difficile d'autofinancer le poste d'une personne ressource engagée pour soutenir le développement d'outils adaptés à la réalité spécifique de l'industrie touristique. Pourtant, les besoins sont là, bien identifiés et gếnéralisés à l'ensemble de l'industrie touristique. Et ces besoins sont confirmés par le diagnostic de l'industrie commandé par le CQRHT et livré en avril 1996.

La création d'un poste permanent spécifique à la formation était, jusqu'à récemment, unique à l'ATR des Cantons-del'Est. Les leçons découlant des problématiques rencontrếes au cours des trois dernières années doivent done être scrutées à la loupe pour nous permettre de faire mieux dans l'avenir.

La formation est encore très marginale apres trois ans de travail. Qu'on parle de besoins de formation en anglais, en accueil, en service à la clientèle ou autre, on a proposé plusieurs projets. Mais on n'a pas encore trouvé la clé pour convaincre les entreprises de notre région qu'il s'agit d'un investissement plutôt que d'une dépense et que les ressources humaines sont la ressource première de l'industrie touristique. En haute saison. tout le monde est occupé donc on n"a pas le temps de former les employés. En basse saison, on n'a pas d'argent pour former les employés qui, pour la plupart, 


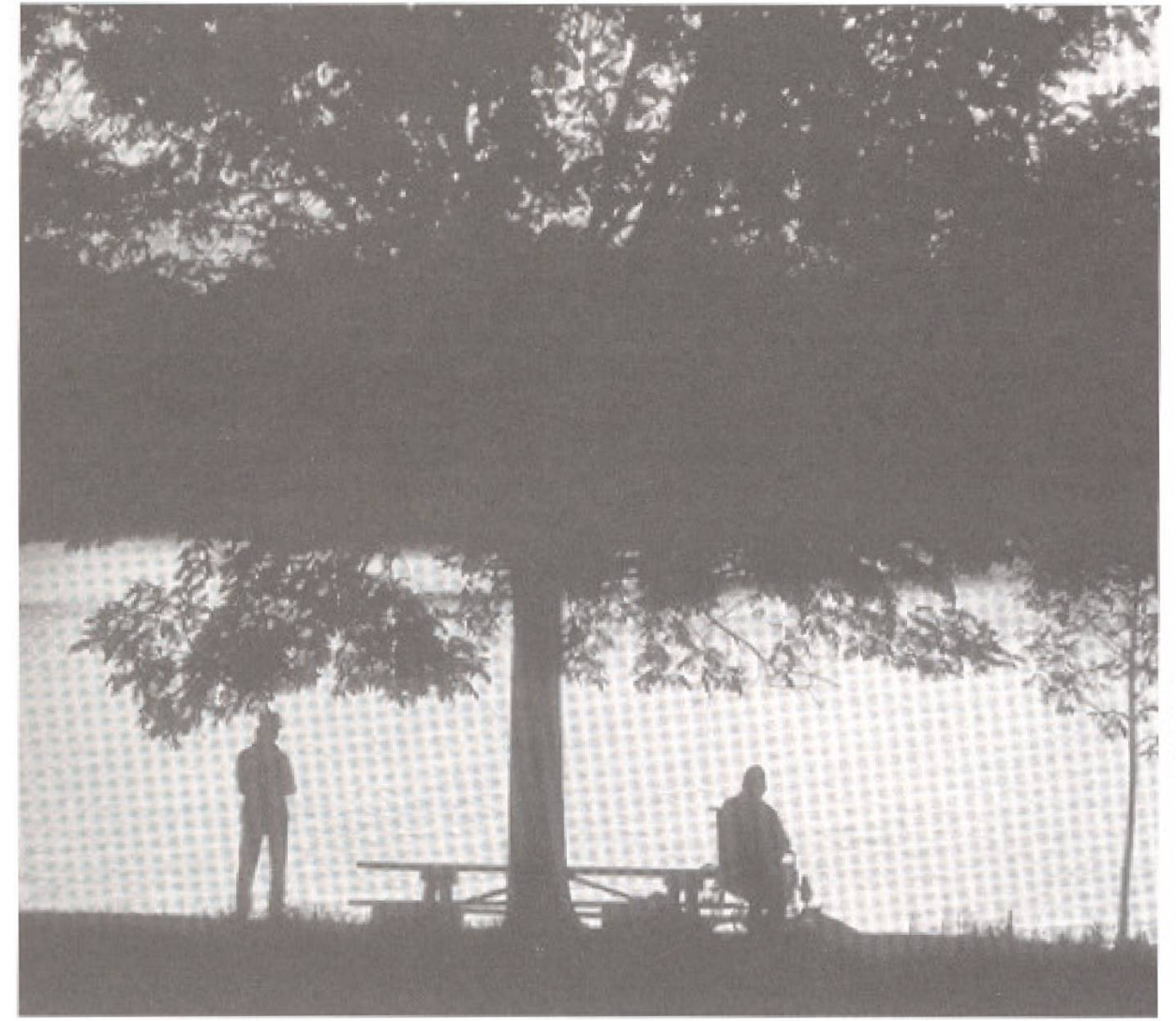

sont mis à pied pour quelques mois. On est donc bien loin du jour où la formation seral considérée comme un investissement.

Avec la Loi 90, toutes les entreprises qui ont une masse salariale de $250000 \$$ et plus seront obligées, d'ici janvier 1998, d'investir $1 \%$ de leur masse salariale en formation. L'ATR, les associations sectorielles, les corporations de développement locales, tous se voient comme percepteur de ces sommes miraculeuses qui leur permettraient de répondre aux besoins en allouant une ressounce pour développer des projets concertés en formation. Mais, les entreprises vont-elles accepter de faire gérer ces montants par un tiers ou préféreront-elles l'administrer' elles-mêmes? À ce jour, peu nombreuses sont celles qui ont accepté une intervention externe pour l'élaboration d'un plan personnalisé de formation. Elles veulent le faire elles-mêmes. Comment alors pouvons-nous imaginer que ces mêmes entreprises verseront à un tiers des sommes qui viseront des intêrêts collectifs plutôt qu'individuels.

L'industrie touristique compte encore bien peu de professionnels formés et compétents pour mener des opérations de formation efficaces au sein de ses entreprises. Elle sera toujours confrontée au phênonène des spedlers qui, comme ils le font déjà depuis plusieurs années en publicité, se présentent et offrent une manne de Kit de formation miracle. Leur budget étant limité, s"ils investissent là, ils n'investiront pas ailleurs. Et adieu! les efforts concertés de développement d'une région ou d'un secteur.

\section{CONCLUSION}

Le travail consenti au cours des trois dernières années à l'ATR des Cantonsde-l'Est est important dans un contexte de changement, Les résultats, si minimes soient-ils, sont perceptibles et doivent servir de base au développement plus hamonieux qui nous attend au cours des prochaines années. Il fallait briser la glace, initier le changement. Eh! bien, c'est fait.

Les regroupements d'entreprises touristiques, qu'ils soient régionaux ou sectoriels, doivent travailler ensemble si on veut des résultats plus probants. A cet effet, le travail du Conseil Québécois des Ressources Humaines en Tourisme sera

déterminant. S'il réussit à établir une structure de partenariat et de mise en réseau pour tout ce qui concerne le développement et la distribution des produits et services développés et si cette structure tient compte des forces et des contraintes de chacun, c'est vers un succès assuré que nous nous dirigeons.

Le momentum est excellent avec l'entrée en vigueur de la Loi 90. Les entreprises ont de l'argent à dépenser en formation. Nous n'avons plus maintenant qu'à travailler ensemble pour leur offrir des produits et services de formation de qualité, qui répondent à leurs besoins et ce, à des prix qu'ils sont capables de payer.

Mais il faut toujours garder à l'esprit que le développement, dans ce domaine comme dans d'autres, est un travail de longue halleine parsemé de petits succès mais aussi de nombreux échecs qui nous obligent à nous remettre en question pour faire mieux la prochaine fois. 